\title{
PARTE 2: PLACAS
}

\section{GENERALIDADES}

Placas são peças estruturais em que uma dimensão, a espessura $e$, é muito menor que as outras duas, são planas, e os carregamentos são supostamente perpendiculares a esse plano, gerando flexão e cisalhamento. É o caso, por exemplo, dos painéis de fechamento de asas de aeronaves, em que pequenas curvaturas permitem a aproximação por um plano e o carregamento predominante são as forças de sustentação sempre normais à superfície da peça. Na Engenharia Civil têm-se as lajes de concreto armado e protendido.

\subsection{Derivando a Equação de Sophie-Germain e Lagrange}

Considere-se a placa da Fig. 2.1, de espessura muito fina $h$, contida no plano horizontal $x-y$. Supõe-se que seja retangular, com comprimento $a$ na direção do eixo $x$ e largura $b$ na direção do eixo $y$. Um carregamento transversal a seu plano (na direção vertical) $p=p(x, y)$ provoca deslocamentos transversais (verticais) de seus pontos, dados por $w=w(x, y)$, conforme Fig. 2.2. Dentro das hipóteses de placas finas de Kirchoff-Love, e adotando-se material elástico linear isotrópico e homogêneo, o problema é regido pela Equação de Sophie-Germain e Lagrange: 


$$
\nabla^{4} w=\frac{p}{D}
$$

onde o operador nabla quatro é

$$
\nabla^{4}=\frac{\partial^{4}}{\partial x^{4}}+2 \frac{\partial^{4}}{\partial x^{2} \partial y^{2}}+\frac{\partial^{4}}{\partial y^{4}}
$$

em que

$$
D=\frac{E e^{3}}{12\left(1-v^{2}\right)}
$$

é a rigidez flexional da placa. Sua dedução é apresentada a seguir.

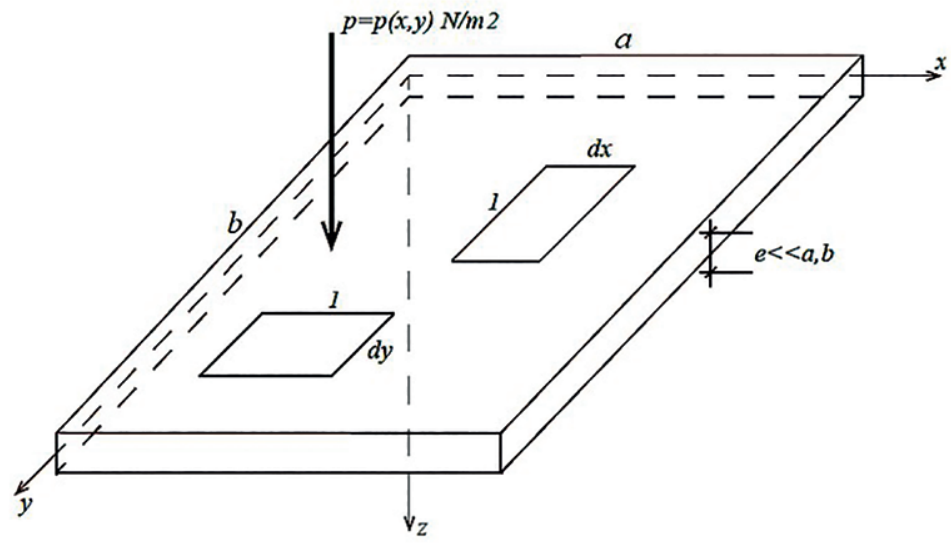

Figura 2.1

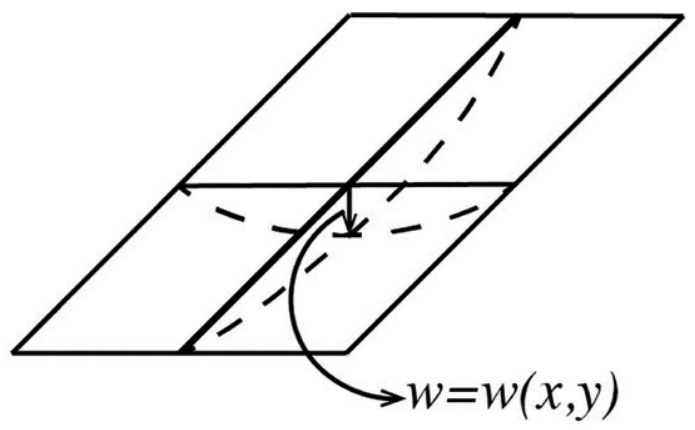

Figura 2.2 


\subsection{Cinemática}

Considere-se, primeiro, um elemento dessa placa de comprimento $d x$ e largura unitária. Adotando-se a hipótese de que as seções permanecem planas após a flexão, conforme Fig. 2.3, tem-se que a deformação de uma fibra distante $z$ do plano neutro é

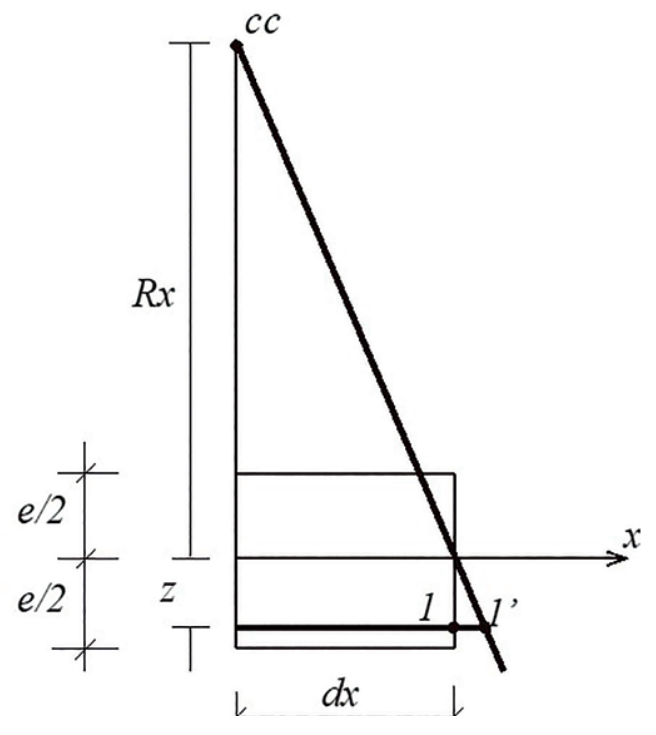

Figura 2.3

$$
\varepsilon_{x}=\frac{11^{\prime}}{d x}=\frac{z}{R_{x}}=\kappa_{x} z
$$

em que

$$
\kappa_{x}=\frac{1}{R_{x}}=-\frac{\partial^{2} w}{\partial x^{2}}
$$

é a curvatura em $x$, o inverso do correspondente raio de curvatura. Considere-se, agora, um elemento dessa placa de comprimento $d y$ e largura unitária. Adotando-se a hipótese de que as seções permanecem planas após a flexão, conforme Fig. 2.4, tem-se que a deformação de uma fibra distante $z$ do plano neutro é 


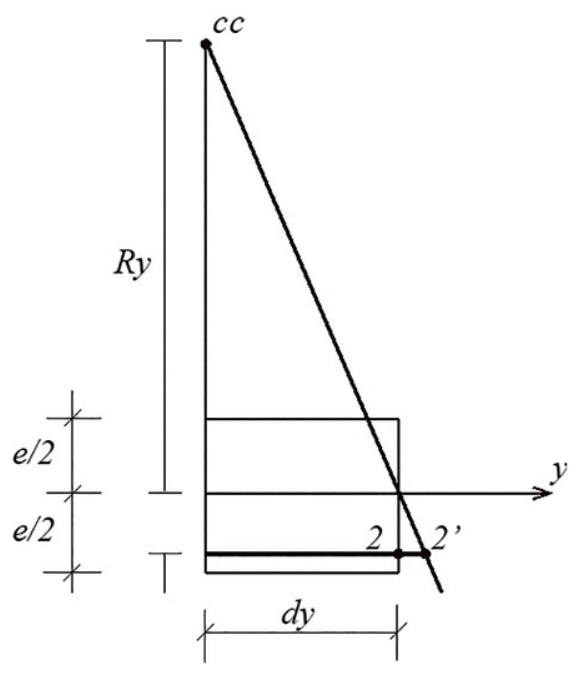

Figura 2.4

$$
\varepsilon_{y}=\frac{22^{\prime}}{d y}=\frac{z}{R_{y}}=\kappa_{y} z
$$

em que

$$
\kappa_{y}=\frac{1}{R_{y}}=-\frac{\partial^{2} w}{\partial y^{2}}
$$

é a curvatura em $y$, o inverso do correspondente raio de curvatura.

\subsection{Lei de Hooke}

Adota-se material elástico linear isotrópico e homogêneo, com módulo de elasticidade $E$ e coeficiente de Poisson $v$. Assim, as tensões normais nas duas direções do plano são

$$
\begin{aligned}
& \sigma_{x}=\frac{E}{1-v^{2}}\left(\varepsilon_{x}+v \varepsilon_{y}\right)=-\frac{E}{1-v^{2}}\left(\frac{\partial^{2} w}{\partial x^{2}}+v \frac{\partial^{2} w}{\partial y^{2}}\right) z \\
& \sigma_{y}=\frac{E}{1-v^{2}}\left(\varepsilon_{y}+v \varepsilon_{x}\right)=-\frac{E}{1-v^{2}}\left(\frac{\partial^{2} w}{\partial y^{2}}+v \frac{\partial^{2} w}{\partial x^{2}}\right) z
\end{aligned}
$$


que atuam como indicado nas Figuras 2.5 e 2.6.

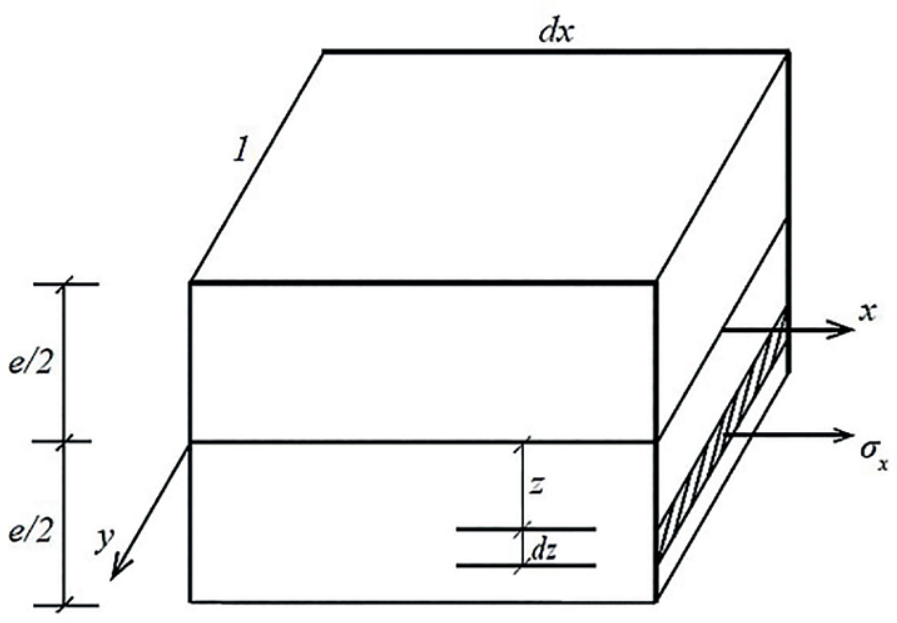

Figura 2.5

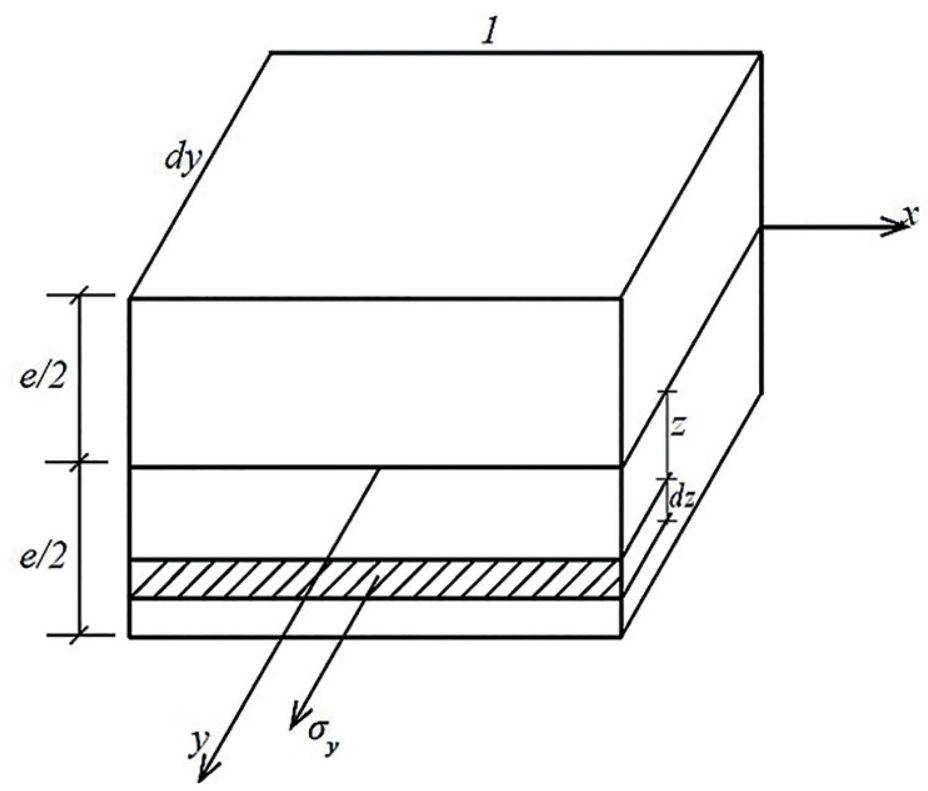

Figura 2.6

A integração dessas tensões fornece os momentos fletores por unidade de comprimento dados por 


$$
\begin{gathered}
M_{x}=\int_{-e / 2}^{e / 2} \sigma_{x} z d z=-D\left(\frac{\partial^{2} w}{\partial x^{2}}+v \frac{\partial^{2} w}{\partial y^{2}}\right) \\
M_{y}=\int_{-e / 2}^{e / 2} \sigma_{y} z d z=-D\left(\frac{\partial^{2} w}{\partial y^{2}}+v \frac{\partial^{2} w}{\partial x^{2}}\right) \\
D=\frac{E}{1-v^{2}} \int_{-e / 2}^{e / 2} z^{2} d z=\frac{E e^{3}}{12\left(1-v^{2}\right)}
\end{gathered}
$$

Tensões tangenciais (de cisalhamento) também atuam nas faces do elemento de placa de comprimentos unitários nas duas direções representado na Fig. 2.7, resultando forças cortantes por unidade de comprimento.

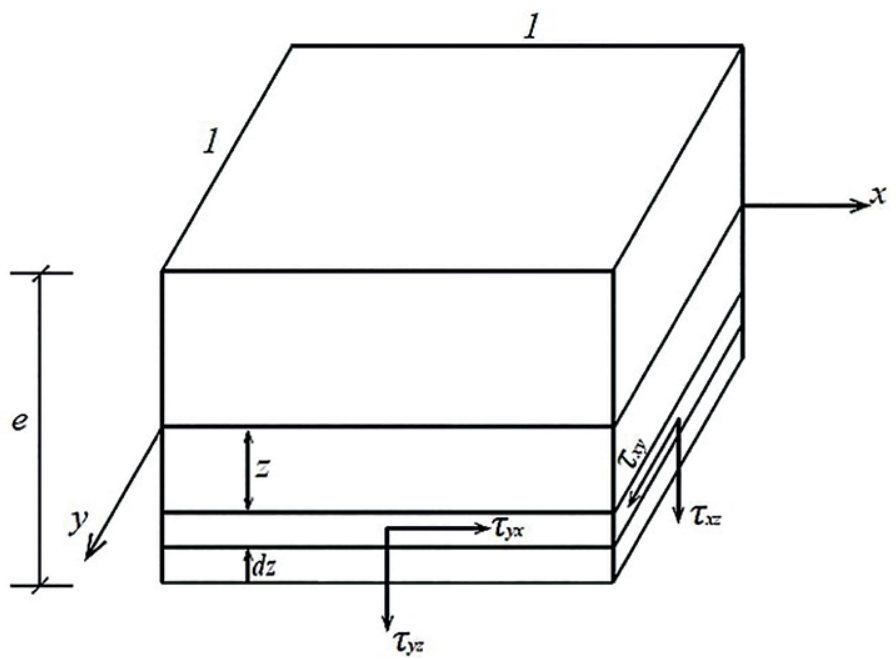

Figura 2.7

$$
Q_{x}=\int_{-e / 2}^{e / 2} \tau_{x z} d z
$$




$$
Q_{y}=\int_{-e / 2}^{e / 2} \tau_{y z} d z
$$

e momentos de torção

$$
\begin{gathered}
M_{x y}=-\int_{-e / 2}^{e / 2} \tau_{x y} z d z=-D\left(1-v^{2}\right) \frac{\partial^{2} w}{\partial x \partial y} \\
M_{x y}=\int_{-e / 2}^{e / 2} \tau_{x y} z d z=D\left(1-v^{2}\right) \frac{\partial^{2} w}{\partial x \partial y}
\end{gathered}
$$

\subsection{Equilíbrio}

Escreve-se, agora, o equilíbrio de forças na vertical de um elemento de placa de comprimentos $d x$ e $d y$ no plano, conforme indicado na Fig. 2.8, resultando

$$
\frac{\partial Q_{x}}{\partial x}+\frac{\partial Q_{y}}{\partial y}=-p
$$

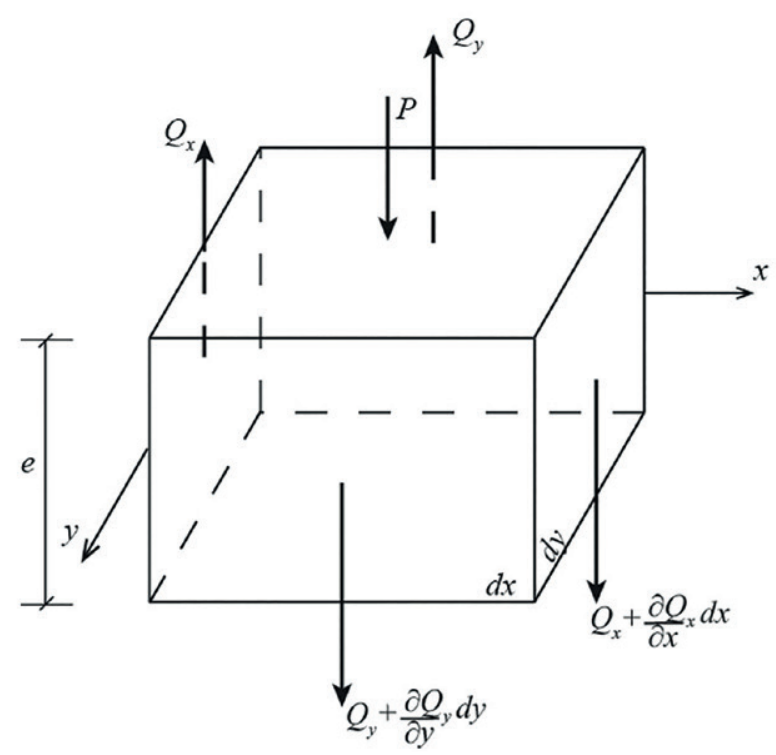

Figura 2.8 


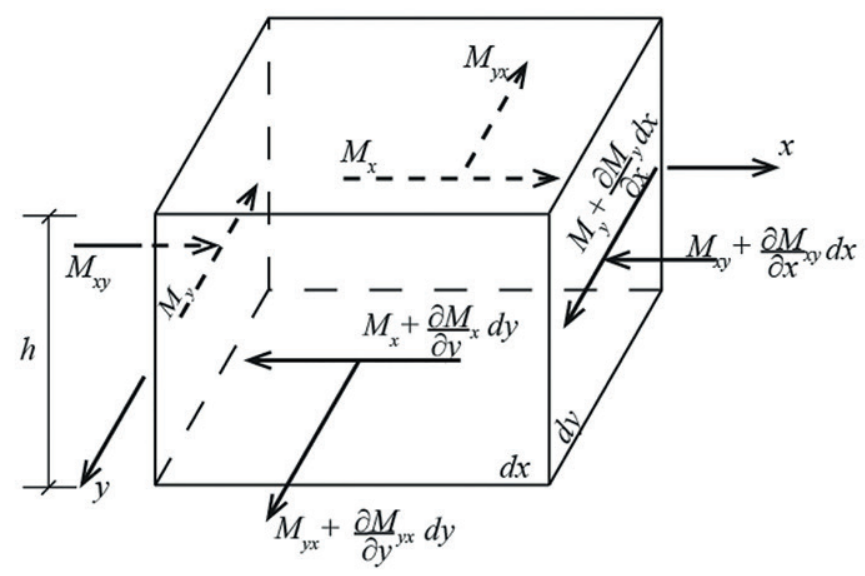

Figura 2.9

Analisando a Fig. 2.9, escreve-se o equilíbrio de momentos em torno dos eixos $x$ e $y$, desprezando-se termos de ordem superior, na forma

$$
\begin{gathered}
Q_{y}=\frac{\partial M_{x}}{\partial y}+\frac{\partial M_{x y}}{\partial x} \\
Q_{x}=\frac{\partial M_{y}}{\partial x}+\frac{\partial M_{y x}}{\partial y}
\end{gathered}
$$

que substituídas na equação de equilíbrio de forças cortantes resulta, finalmente,

$$
\frac{\partial^{2} M_{y}}{\partial x^{2}}+2 \frac{\partial^{2} M_{x y}}{\partial x \partial y}+\frac{\partial^{2} M_{x}}{\partial y^{2}}=-p
$$

Agora, basta substituir as expressões dos momentos fletores e de torção, escritas em função do deslocamento transversal $w$ para chegar à já apresentada Equação de Sophie-Germain e Lagrange

$$
\nabla^{4} w=\frac{p}{D}
$$




\subsection{Solução}

Se for determinada a função $w=w(x, y)$, pode-se, em princípio, determinar os esforços solicitantes na placa. Os momentos fletores, por unidade de comprimento, já apresentados, são

$$
\begin{aligned}
& M_{y}=-D\left(\frac{\partial^{2} w}{\partial y^{2}}+v \frac{\partial^{2} w}{\partial x^{2}}\right) \\
& M_{x}=-D\left(\frac{\partial^{2} w}{\partial x^{2}}+v \frac{\partial^{2} w}{\partial y^{2}}\right)
\end{aligned}
$$

Os momentos de torção, por unidade de comprimento, são

$$
\begin{gathered}
M_{x y}=-D(1-v) \frac{\partial^{2} w}{\partial x \partial y} \\
M_{y x}=D(1-v) \frac{\partial^{2} w}{\partial x \partial y}
\end{gathered}
$$

e as forças cortantes, também por unidade de comprimento, já apresentadas, são

$$
\begin{aligned}
& Q_{x}=\frac{\partial M_{y}}{\partial x}+\frac{\partial M_{x y}}{\partial y} \\
& Q_{y}=\frac{\partial M_{x}}{\partial y}+\frac{\partial M_{y x}}{\partial x}
\end{aligned}
$$

Trata-se de uma equação diferencial parcial de quarta ordem cuja solução depende das condições de contorno nos lados da placa. No geral:

1. simplesmente apoiada, em que o deslocamento vertical e a derivada segunda, relacionada com o momento fletor, são nulos;

2. engastada, em que o deslocamento vertical e a derivada primeira, relacionada com a tangente à superfície deformada, são nulos;

3. livre, em que o deslocamento é livre e os momentos fletores são nulos. 
Na prática, soluções fechadas são muito difíceis de se obter. Com a disponibilidade atual de métodos computacionais, esses são preferíveis para fins profissionais e serão detalhados na sequência deste capítulo. Existem na literatura técnica, entretanto, soluções obtidas por séries trigonométricas infinitas, uma vez que funções harmônicas são tratáveis quando aplicadas à Equação de Sophie-Germain e Lagrange e a condições de contorno usuais. Tabelas geradas por essas soluções podem ser encontradas em, por exemplo, Timoshenko.

\section{DINÂMICA EM PLACAS}

\subsection{Carregamento genérico}

No caso de uma análise dinâmica, tanto o carregamento como os deslocamentos resultantes são funções também do tempo, isto é, $p=p(x, y, t) \mathrm{e}$ $w=w(x, y, t)$. Além disso, de acordo com a segunda lei de Newton, surgem as forças de inércia, massa vezes aceleração. Assim, a equação de Sophie-Germain e Lagrange se escreve

$$
\nabla^{4} w=\frac{1}{D}(p+\rho \ddot{w})
$$

em que $\rho$ é a densidade do material $\left(\mathrm{kg} / \mathrm{m}^{3}\right)$, suposta constante, e

$$
\ddot{w}=\frac{\partial^{2} w}{\partial t^{2}}
$$

No caso de vibrações livres amortecidas, o único carregamento externo é a força dissipativa. Se for suposta do tipo viscosa, proporcional à velocidade, tem-se

$$
p=c \dot{w}=c \frac{\partial w}{\partial t}
$$

em que $c$ é uma constante de amortecimento viscoso, obtida experimentalmente ou adotada por indicações de normas.

\subsection{Vibrações livres não amortecidas}

A equação do movimento para vibrações livres não amortecidas de placas finas é 


$$
D \nabla^{4} w+\rho \frac{\partial^{2} w}{\partial t^{2}}=0
$$

Neste caso de vibrações livres, o vetor de deslocamentos, nos nós de coordenadas $x$ e $y$ de uma malha de pontos resultante de uma discretização do contínuo no interior da placa, varia harmonicamente com o tempo, na forma

$$
\{w(x, y, t)\}=\{W(x, y)\} \cos \omega t
$$

onde $\omega$ é uma frequência circular de vibração (em radianos/segundo), e $\{W(x, y)\}$ um vetor função apenas das coordenadas espaciais, também conhecido como modo de vibração, levando à equação do movimento

$$
\left(\nabla^{4}-\lambda\right)\{W\}=0
$$

onde

$$
\lambda=\frac{\rho \omega^{2}}{D}
$$

Temos, assim, um problema de autovalores e autovetores.

\begin{tabular}{|c|c|c|c|}
\hline $\begin{array}{l}\text { CONDIÇÕES DE } \\
\text { CONTORNO }\end{array}$ & FUNÇÃO DE DEFLEXÃO & $\mathbf{N}$ & $\mathbf{K}$ \\
\hline 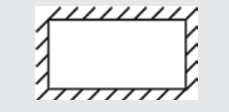 & $\left(\cos \frac{2 \pi x}{a}-1\right)\left(\cos \frac{2 \pi y}{b}-1\right)$ & 2.25 & $12+8\left(\frac{a}{b}\right)^{2}+12\left(\frac{a}{b}\right)^{4}$ \\
\hline 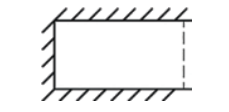 & $\left(\cos \frac{3 \pi x}{2 a}-\cos \frac{\pi x}{2 a}\right)\left(\cos \frac{2 \pi y}{b}-1\right)$ & 1.50 & $3.85+5\left(\frac{a}{b}\right)^{2}+8\left(\frac{a}{b}\right)^{4}$ \\
\hline 兵 & $\left(1-\cos \frac{\pi x}{2 a}\right)\left(\cos \frac{2 \pi y}{b}-1\right)$ & .340 & $0.0468+0.340\left(\frac{a}{b}\right)^{2}+1.814\left(\frac{a}{b}\right)^{4}$ \\
\hline 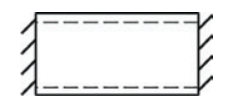 & $\left(\cos \frac{2 \pi x}{a}-1\right) \sin \frac{\pi y}{b}$ & .75 & $4+2\left(\frac{a}{b}\right)^{2}+0.75\left(\frac{a}{b}\right)^{4}$ \\
\hline
\end{tabular}

Soluções fechadas, obtidas por séries trigonométricas, para as frequências de placas podem ser encontradas na publicação da NASA de autoria do Prof. A. Leissa. 


\begin{tabular}{|c|c|c|c|}
\hline$\lambda_{-\ldots \ldots \ldots}$ & $\left(\cos \frac{2 \pi x}{a}-1\right) \frac{y}{b}$ & .50 & $2.67+0.304\left(\frac{a}{b}\right)^{2}$ \\
\hline$\lambda^{\lambda}$ & $\cos \frac{2 \pi x}{a}-1$ & 1.5 & 8 \\
\hline ג & $\left(\cos \frac{3 \pi x}{2 a}-\cos \frac{\pi x}{2 a}\right)\left(\cos \frac{3 \pi y}{2 b}-\cos \frac{\pi y}{2 b}\right)$ & 1.00 & $2.56+3.12\left(\frac{a}{b}\right)^{2}+2.56\left(\frac{a}{b}\right)^{4}$ \\
\hline 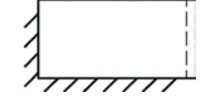 & $\left(\cos \frac{3 \pi x}{2 a}-\cos \frac{\pi x}{2 a}\right)\left(1-\cos \frac{\pi y}{2 b}\right)$ & .227 & $0.581+0.213\left(\frac{a}{b}\right)^{2}+0.031\left(\frac{a}{b}\right)^{4}$ \\
\hline 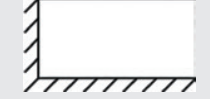 & $\left(1-\cos \frac{\pi x}{2 a}\right)\left(1-\cos \frac{\pi y}{2 b}\right)$ & 0.0514 & $0.0072+0.024\left(\frac{a}{b}\right)^{2}$ \\
\hline & $\left(\cos \frac{3 \pi x}{2 a}-\cos \frac{\pi x}{2 a}\right) \sin \frac{\pi y}{b}$ & .50 & $1.28+1.25\left(\frac{a}{b}\right)^{2}+0.5\left(\frac{a}{b}\right)^{4}$ \\
\hline 久. & $\left(\cos \frac{3 \pi x}{2 a}-\cos \frac{\pi x}{2 a}\right) \frac{y}{b}$ & .333 & $0.853+0.190\left(\frac{a}{b}\right)^{2}$ \\
\hline$\lambda$ & $\cos \frac{3 \pi x}{2 a}-\cos \frac{\pi x}{2 a}$ & 1.00 & 2.56 \\
\hline$\hat{\gamma}_{------}$ & $\left(1-\cos \frac{\pi x}{2 a}\right) \frac{\pi^{2}}{b^{2}} \sin \frac{\pi y}{b}$ & .1134 & $0.0156+0.0852\left(\frac{a}{b}\right)^{2}$ \\
\hline$\lambda_{-}$ & $\left(1-\cos \frac{\pi x}{2 a}\right) \frac{y}{b}$ & .0756 & $0.0104+0.0190\left(\frac{a}{b}\right)^{2}$ \\
\hline 久 & $1-\cos \frac{\pi x}{2 a}$ & 2268 & 0.0313 \\
\hline & $\sin \frac{\pi x}{a} \sin \frac{\pi y}{b}$ & .25 & $0.25+0.50\left(\frac{a}{b}\right)^{2}+0.25\left(\frac{a}{b}\right)^{4}$ \\
\hline ! & $\left(\sin \frac{\pi x}{a}\right) \frac{y}{b}$ & .1667 & $0.1667+0.0760\left(\frac{a}{b}\right)^{2}$ \\
\hline i & $\sin \frac{\pi x}{a}$ & .50 & 0.50 \\
\hline
\end{tabular}


Por exemplo, para uma placa retangular simplesmente apoiada em todo seu perímetro, calcula-se a rigidez flexional

$$
D=\frac{E e^{3}}{12\left(1-v^{2}\right)}
$$

e coeficientes $K$

$$
K=0.25+0.5\left(\frac{a}{b}\right)^{2}+0.25\left(\frac{a}{b}\right)^{4}
$$

e $N=0.2$, dados na Tabela para esta condição de contorno. A frequência correspondente, dada em rad/s, é

$$
\omega=2\left(\frac{\pi}{a}\right)^{2} \sqrt{\frac{D K}{\rho N}}
$$

Uma solução numérica muito conveniente é obtida pela aplicação do Método das Diferenças Finitas, como se verá na seção 4. desta Parte 2.

\section{ESTABILIDADE: EQUAÇ̃̃O DE SAINTTVENANT}

Considere-se, agora o caso de presença de forças normais $N_{x}$ e $N_{y}$, e de cisalhamento $N_{x y}$ e $N_{y x}$, por unidade de comprimento, contidas no plano $x y$ da placa, conforme Fig. 2.10. Se forem de pequena intensidade, espera-se que não influenciem nos deslocamentos transversais $w$ da placa. Caso contrário, isso pode ocorrer, e levar, no limite, à instabilidade (flambagem). A projeção dessas forças no plano $x y$ da placa resultam as equações de equilíbrio:

$$
\begin{aligned}
& \frac{\partial N_{x}}{\partial x}+\frac{\partial N_{y x}}{\partial y}=0 \\
& \frac{\partial N_{y}}{\partial y}+\frac{\partial N_{x y}}{\partial x}=0
\end{aligned}
$$


Essas equações são inteiramente independentes das 3 equações de equilíbrio deduzidas na seção 1.4 desta Parte 2.

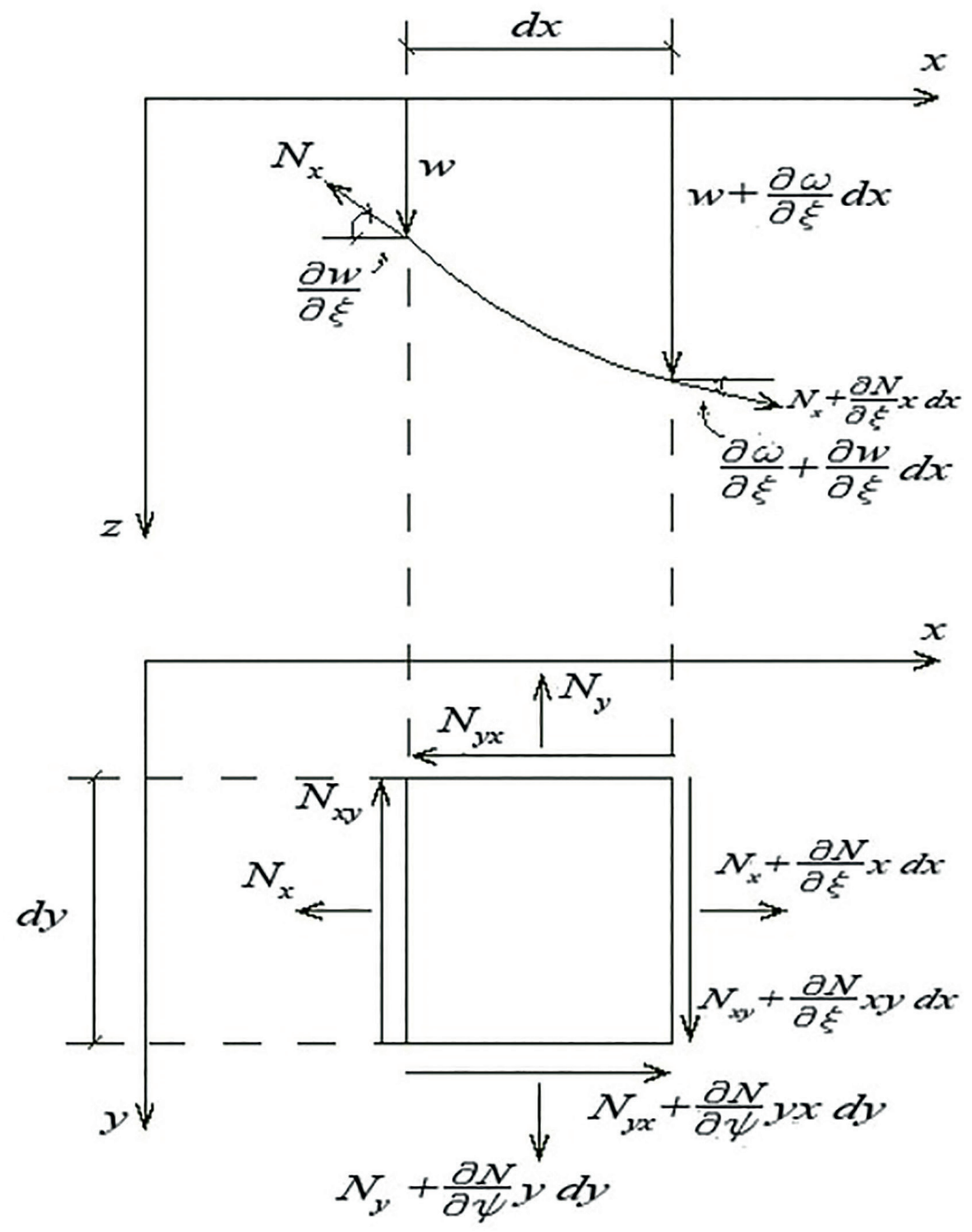

Figura 2.10

Agora considere-se a projeção das forças normais no plano $x z$ na direção $z$, a direção transversal ao plano da placa, levando em conta, como mostrado na Fig. 2.10, o deslocamento transversal $w$ de seu plano neutro. Resulta

$$
\frac{\partial N_{y}}{\partial y}+\frac{\partial N_{x y}}{\partial x}=0
$$




$$
-N_{x} d y \frac{\partial w}{\partial x} d x d y+\left(N_{x}+\frac{\partial N_{x}}{\partial x} d x\right)\left(\frac{\partial w}{\partial x}+\frac{\partial^{2} w}{\partial x^{2}} d x\right) d y
$$

Simplificando e desconsiderando termos de ordem superior, tem-se

$$
N_{x} \frac{\partial^{2} w}{\partial x^{2}} d x d y+\frac{\partial N_{x}}{\partial x} \frac{\partial w}{\partial x} d x d y
$$

Da mesma forma, considere-se a projeção das forças normais no plano $y z$ na direção $z$, levando a

$$
N_{y} \frac{\partial^{2} w}{\partial y^{2}} d x d y+\frac{\partial N_{y}}{\partial y} \frac{\partial w}{\partial y} d x d y
$$

Um raciocínio bem mais elaborado, aqui omitido, é necessário para obtenção da projeção das forças de cisalhamento na direção do eixo $z$, levando a

$$
2 N_{x y} \frac{\partial^{2} w}{\partial x \partial y} d x d y+\frac{\partial N_{x y}}{\partial x} \frac{\partial w}{\partial y} d x d y+\frac{\partial N_{x y}}{\partial y} \frac{\partial w}{\partial x} d x d y
$$

A seguir, todas essas componentes de forças na direção $z$ serão somadas às forças nessas direções já consideradas no equilíbrio de forças verticais determinado na seção 1.4 desta Parte 2, levando a uma nova forma da Equação de Sophie-Germain e Lagrange, primeiramente obtida por Saint-Venant, na forma:

$$
\nabla^{4} w=\frac{1}{D}\left(p+N_{x} \frac{\partial^{2} w}{\partial x^{2}}+N_{y} \frac{\partial^{2} w}{\partial y^{2}}+2 N_{x y} \frac{\partial^{2} w}{\partial x \partial y}\right)
$$

No caso particular de carregamento transversal $p$ nulo, tem-se a formulação teórica necessária à determinação do carregamento que levaria à instabilidade (flambagem) de uma placa fina.

Mais uma vez, na prática, soluções fechadas são muito difíceis de se obter. Com a disponibilidade atual de métodos computacionais, esses são preferíveis para fins profissionais. Existem na literatura técnica, entretanto, soluções obtidas por séries trigonométricas infinitas, uma vez que funções harmônicas são tratáveis quando aplicadas à Equação de Sophie-Germain e Lagrange e a condições de contorno usuais. Tabelas geradas por essas soluções podem ser encontradas em, por exemplo, Leissa. 


\section{MÉTODO DAS DIFERENCASS FINITAS EM PLACAS}

\subsection{Generalidades}

A finalidade dos métodos de discretização é reduzir um problema contínuo a um sistema com um número finito de graus de liberdade. No geral, tem-se um fenômeno físico governado por uma equação diferencial, ou um sistema delas, envolvendo uma ou mais funções. A solução dessas equações, a obtenção da expressão da função em forma fechada quase nunca é possível, e uma boa aproximação pode ser obtida de um número limitado de parâmetros se o analista tiver boa sensibilidade.

A aproximação basicamente envolve trocar um domínio contínuo por uma malha de pontos discretos no interior desse domínio. Em vez de se obter a função contínua nesse domínio, obtém-se aproximações para o valor que essa função assume somente em cada um desses pontos isolados.

No caso em estudo, das placas, a equação diferencial governante no domínio da placa, já vista, é a de Sophie-Germain e Lagrange:

$$
\nabla^{4} w=\frac{p}{D}
$$

onde o operador nabla quatro é

$$
\nabla^{4}=\frac{\partial^{4}}{\partial x^{4}}+2 \frac{\partial^{4}}{\partial x^{2} \partial y^{2}}+\frac{\partial^{4}}{\partial y^{4}}
$$

Além disso, há que impor as condições de contorno nas faces da placa. Por exemplo, se for um apoio simples, os momentos fletores são nulos e, portanto, a derivada segunda é zero nessa extremidade. Já uma face engastada implica em que a tangente à deformada ali é nula, ou seja, a derivada primeira é zero.

No Método das Diferenças Finitas, as diversas derivadas presentes nas equações diferenciais no domínio e nas condições de contorno, são aproximadas por expressões de diferenças, em geral obtidas de séries de Taylor. O procedimento foi primeiro aplicado a domínios bidimensionais, como neste caso, por C. Runge, em 1908.

As expressões necessárias para o propósito deste capítulo são apresentadas a seguir, para um ponto pertencente a uma linha $j$ e uma coluna $k$, de uma malha retangular com espaçamento $h_{x}$ na direção do eixo $x$ e $h_{y}$ na do $y$. 


$$
\begin{aligned}
& \left(\frac{\partial w}{\partial x}\right)_{j, k}=\frac{w_{j, k+1}-w_{j, k-1}}{2 h_{x}}\left(\frac{\partial w}{\partial y}\right)_{j, k}=\frac{w_{j+1, k}-w_{j-1, k}}{2 h_{y}} \\
& \left(\frac{\partial^{2} w}{\partial x^{2}}\right)_{j, k}=\frac{w_{j, k+1}-2 w_{j, k}+w_{j, k-1}}{h_{x}^{2}} \\
& \left(\frac{\partial^{2} w}{\partial y^{2}}\right)_{j, k}=\frac{w_{j+1, k}-2 w_{j, k}+w_{j-1, k}}{h_{y}^{2}} \\
& \left(\frac{\partial^{4} w}{\partial x^{4}}\right)_{j, k}=\frac{w_{j, k+2}-4 w_{j, k+1}+6 w_{j, k}-4 w_{j, k-1}+w_{j, k-2}}{h_{x}^{4}} \\
& \left(\frac{\partial^{4} w}{\partial y^{4}}\right)_{j, k}=\frac{w_{j+2, k}-4 w_{j+1, k}+6 w_{j, k}-4 w_{j-1, k}+w_{j-2, k}}{h_{y}{ }^{4}} \\
& \left(\frac{\partial^{4} w}{\partial x^{2} \partial y^{2}}\right)_{j, k}=\frac{1}{h_{x}{ }^{2} h_{y}{ }^{2}}\left(w_{j+1, k-1}-2 w_{j+1, k}+w_{j+1, k+1}-2 w_{j, k-1}\right. \\
& \left.+4 w_{j, k}-2 w_{j, k+1}+w_{j-1, k-1}-2 w_{j-1, k}+w_{j-1, k+1}\right)
\end{aligned}
$$

\subsection{Exemplo básico}

Considere-se uma placa simplesmente apoiada em todo perímetro, de planta quadrada $2 \times 2 \mathrm{~m}$, espessura $e=2 \mathrm{~cm}$, carregada de cima para baixo por carga uniformemente distribuída $p=2 \mathrm{KPa}$, módulo de elasticidade $E=200 \mathrm{GPa}$ e coeficiente de Poisson $v=0,3$.

$$
D=\frac{E e^{3}}{12\left(1-v^{2}\right)}=146,52
$$


Para comparação, será calculado o deslocamento vertical máximo, no meio da placa, para duas malhas, uma com $h_{x}=h_{y}=1 \mathrm{~m}$ e a outra $h_{x}=h_{y}=0,5 \mathrm{~m}$.

Para a malha mais grosseira, tem-se apenas um ponto no interior do domínio, e a aplicação da equação diferencial em sua forma de diferenças finitas necessita que se considere pontos externos ao domínio.

Nas linhas do contorno apoiado, os deslocamentos verticais são zero. Também o momento fletor (derivada segunda) é nulo, implicando que os deslocamentos dos pontos externos são iguais a menos o deslocamento do ponto interno. Com isso, chega-se a

$$
16 w_{0,0}=\frac{2}{146}, 52=0,01365 w_{0,0}=0,8531 \mathrm{~mm}
$$

Para a malha mais fina tem-se nove pontos no interior do domínio. Por razões de simetria, basta fazer a aplicação da equação diferencial em sua forma de diferenças finitas em apenas três desses pontos, no primeiro quadrante.

Ponto 0,0

$$
\begin{aligned}
w_{0,2}-8 w_{0,1}+ & 20 w_{0,0}-8 w_{0,-1}+w_{0,-2}+w_{2,0}-8 w_{1,0}-8 w_{-1,0}+w_{-2,0} \\
& +2\left(w_{1,1}+w_{1,-1}+w_{-1,1}+w_{-1,-1}\right)=0,5^{4} \frac{p}{D}
\end{aligned}
$$

\section{Ponto 0,1}

$$
\begin{aligned}
w_{0,3}-8 w_{0,2}+ & 20 w_{0,1}-8 w_{0,0}+w_{0,-1}+w_{2,1}-8 w_{1,1}-8 w_{-1,1}+w_{-2,1} \\
& +2\left(w_{1,2}+w_{1,0}+w_{-1,2}+w_{-1,0}\right)=0,5^{4} \frac{p}{D}
\end{aligned}
$$

\section{Ponto 1,1}

$$
\begin{gathered}
w_{1,3}-8 w_{1,2}+20 w_{1,1}-8 w_{1,0}+w_{1,-1}+w_{3,1}-8 w_{2,1}-8 w_{0,1}+w_{-1,1} \\
+2\left(w_{2,2}+w_{2,0}+w_{0,2}+w_{0,0}\right)=0,5^{4} \frac{p}{D}
\end{gathered}
$$

Levando em conta as condições de contorno e de simetria, chega-se ao sistema algébrico linear de 3 equações em 3 incógnitas 


$$
\left[\begin{array}{ccc}
20 & -32 & 8 \\
-8 & 24 & -16 \\
2 & -16 & 20
\end{array}\right]\left\{\begin{array}{l}
w_{0,0} \\
w_{0,1} \\
w_{1,1}
\end{array}\right\}=0,5^{4} x 0,01365\left\{\begin{array}{l}
1 \\
1 \\
1
\end{array}\right\}
$$

Para comparação, o resultado obtido por Timoshenko por séries trigonométricas é $\boldsymbol{w}_{\mathbf{0 , 0}}=0,8867 \mathrm{~mm}$

\subsection{Exemplo mais completo}

Considere-se a placa da Fig. 2.11 simplesmente apoiada em duas bordas paralelas a $x$ e engastada nas outras duas, de planta retangular $8 \times 4 \mathrm{~m}$, espessura $e=5 \mathrm{~cm}$, carregada de cima para baixo por carga uniformemente distribuída $p=100 \mathrm{~Pa}$, módulo de elasticidade $E=3 \mathrm{GPa}$ e coeficiente de Poisson $v=0,25$.

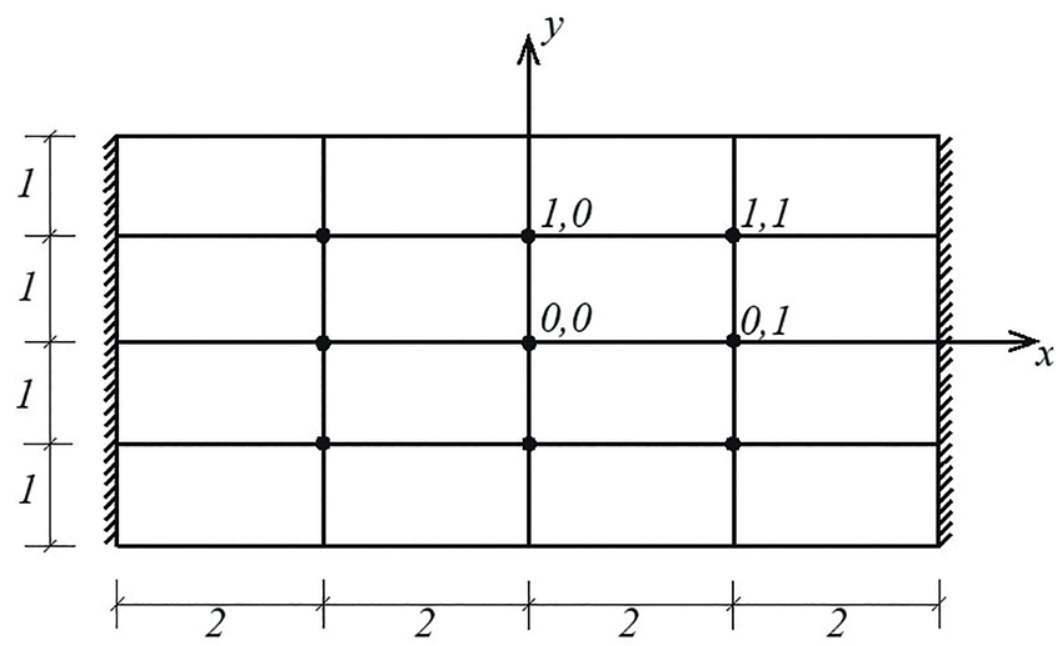

Figura 2.11

Pelas simetrias observáveis na figura, basta aplicar a equação diferencial nos 4 pontos indicados no primeiro quadrante, dos 9 pontos internos. 


\section{Ponto 0,0}

$$
\begin{aligned}
& \frac{1}{16}\left(w_{0,2}-4 w_{0,1}+6 w_{0,0}-4 w_{0,-1}+w_{0,-2}\right) \\
& +\frac{1}{1}\left(w_{2,0}-4 w_{1,0}+6 w_{0,0}-4 w_{-1,0}+w_{-2,0}\right) \\
& +\frac{2}{4}\left(w_{1,1}-2 w_{1,0}+w_{1,-1}-2 w_{0,1}+4 w_{0.0}-2 w_{0,-1}+w_{-1,1}\right. \\
& \left.-2 w_{-1,0}+w_{-1,-1}\right)=\frac{p}{D}=0,003
\end{aligned}
$$

\section{Ponto 0,1}

$$
\begin{aligned}
& \frac{1}{16}\left(w_{0,3}-4 w_{0,2}+6 w_{0,1}-4 w_{0,0}+w_{0,-1}\right) \\
& +\frac{1}{1}\left(w_{2,1}-4 w_{1,1}+6 w_{0,1}-4 w_{-1,1}+w_{-2,1}\right) \\
& +\frac{2}{4}\left(w_{1,2}-2 w_{1,1}+w_{1,0}-2 w_{0,2}+4 w_{0.1}-2 w_{0,0}+w_{-1,2}-2 w_{-1,1}\right. \\
& \left.+w_{-1,0}\right)=\frac{p}{D}=0,003
\end{aligned}
$$

\section{Ponto 1,0}

$$
\begin{aligned}
& \frac{1}{16}\left(w_{1,2}-4 w_{1,1}+6 w_{1,0}-4 w_{1,-1}+w_{1,-2}\right) \\
& +\frac{1}{1}\left(w_{3,0}-4 w_{2,0}+6 w_{1,0}-4 w_{0,0}+w_{-1,0}\right) \\
& +\frac{2}{4}\left(w_{2,1}-2 w_{2,0}+w_{2,-1}-2 w_{1,1}+4 w_{1.0}-2 w_{1,-1}+w_{0,1}-2 w_{0,0}\right. \\
& \left.+w_{0,-1}\right)=\frac{p}{D}=0,003
\end{aligned}
$$




\section{Ponto 1,1}

$$
\begin{aligned}
& \frac{1}{16}\left(w_{1,3}-4 w_{1,2}+6 w_{1,1}-4 w_{1,0}+w_{1,-1}\right) \\
& +\frac{1}{1}\left(w_{3,1}-4 w_{2,1}+6 w_{1,1}-4 w_{0,1}+w_{-1,1}\right) \\
& +\frac{2}{4}\left(w_{2,2}-2 w_{2,1}+w_{2,0}-2 w_{1,2}+4 w_{1.1}-2 w_{1,0}+w_{0,2}-2 w_{0,1}\right. \\
& \left.+w_{0,0}\right)=\frac{p}{D}=0,003
\end{aligned}
$$

Levando em conta as condições de contorno e de simetria, chega-se ao sistema algébrico linear de 4 equações em 4 incógnitas

$$
\left[\begin{array}{cccc}
8,375 & -2,5 & -10 & 2 \\
-1,25 & 8,5 & 1 & -10 \\
-5 & 1 & 8,375 & -2,5 \\
0,5 & -5 & -1,25 & 8,5
\end{array}\right]\left\{\begin{array}{l}
w_{0,0} \\
w_{0,1} \\
w_{1,0} \\
w_{1,1}
\end{array}\right\}=0,003\left\{\begin{array}{l}
1 \\
1 \\
1 \\
1
\end{array}\right\}
$$

cuja solução é

$$
\left\{\begin{array}{l}
w_{0,0} \\
w_{0,1} \\
w_{1,0} \\
w_{1,1}
\end{array}\right\}=0,001\left\{\begin{array}{l}
7,097 \\
5,204 \\
5,012 \\
3,745
\end{array}\right\} m
$$

\subsection{Vibrações livres não amortecidas}

A análise de vibrações livres de placas delgadas discretizadas pelo Método das Diferenças Finitas parte da instituição de um vetor $w$ de deslocamentos verticais dos $n$ nós da malha de diferenças finitas:

$$
\{w(x, y, t)\}=\left\{\begin{array}{c}
w_{1} \\
\cdots \\
w_{n}
\end{array}\right\}
$$

Esse vetor pode ser considerado como o produto de um vetor $\{W(x, y)\}$, também com $n$ componentes, invariável no tempo, ou seja, função apenas de $x$ 
e de $y$, chamado modo de vibração, por uma função do tempo, que supomos ser harmônica, com frequência $\omega$ :

$$
\{w\}=\{W\} \cos \omega
$$

Substituindo-se a solução (2.53) na equação do movimento para vibrações livres, e considerando-se que os deslocamentos $w$ foram obtidos por aproximações de diferenças finitas, chega-se ao problema de autovalores e autovetores:

$$
([A]-\lambda[I])\{W\}=0
$$

em que

$$
\lambda=\frac{\rho \omega^{2}}{D}
$$

As expressões necessárias para escrever os termos gerais dos coeficientes dessa matriz $[A]$ são apresentadas a seguir, tendo por base em um ponto centrado em uma linha $j$ e uma coluna $k$, de uma malha retangular com espaçamento $h_{x}$ na direção do eixo $x$ e $h_{y}$ na do $y$. Esses coeficientes devem ser superpostos com os gerados nos pontos vizinhos a esse. Além disso, as condições de contorno têm que ser impostas. Os detalhes computacionais para essas duas operações são aqui omitidos pela dificuldade de serem detalhados com a clareza necessária, mas o algoritmo completo e testado, em linguagem MATLAB, é dado no anexo deste livro.

$$
\begin{aligned}
& A(j, k)=\frac{6}{h_{x}^{4}}+\frac{6}{h_{y}^{4}}+\frac{8}{h_{x}^{2} h_{y}^{2}} \\
& A(j, k+1)=-\frac{4}{h_{x}^{4}}-\frac{4}{h_{x}^{2} h_{y}^{2}} \\
& A(j, k-1)=-\frac{4}{h_{x}^{4}}-\frac{4}{h_{x}^{2} h_{y}^{2}}
\end{aligned}
$$




$$
\begin{aligned}
& A(j+1, k)=-\frac{4}{h_{y}^{4}}-\frac{4}{h_{x}^{2} h_{y}^{2}} \\
& A(j-1, k)=-\frac{4}{h_{y}^{4}}-\frac{4}{h_{x}^{2} h_{y}^{2}} \\
& A(j, k+2)=\frac{1}{h_{x}^{4}} \\
& A(j, k-2)=\frac{1}{h_{x}^{4}} \\
& A(j+2, k)=\frac{1}{h_{y}^{4}} \\
& A(j-2, k)=\frac{1}{h_{y}^{4}} \\
& A(j+1, k+1)=\frac{2}{h_{x}^{2} h_{y}^{2}} \\
& A(j-1, k+1)=\frac{2}{h_{x}^{2} h_{y}^{2}} \\
& A(j+1, k-1)=\frac{2}{h_{x}^{2} h_{y}^{2}} \\
& A(j-1, k-1)=\frac{2}{h_{x}^{2} h_{y}^{2}}
\end{aligned}
$$




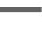

